Radiologe 2021 · 61:1057-1059

https://doi.org/10.1007/s00117-021-00936-x

Angenommen: 2. November 2021

(C) The Author(s), under exclusive licence to Springer Medizin Verlag GmbH, ein Teil von Springer Nature 2021

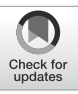

\section{Systemische Skeletterkrankungen}

\author{
Marc-André Weber \\ Institut für Diagnostische und Interventionelle Radiologie, Kinder- und Neuroradiologie, \\ Universitätsmedizin Rostock, Rostock, Deutschland
}
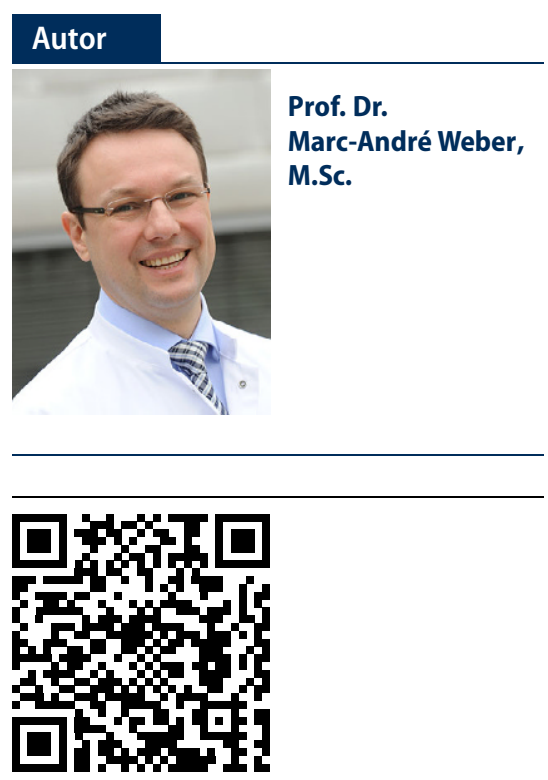

QR-Code scannen \& Beitrag online lesen
Liebe Leserinnen und Leser,

dieses ist für mich ein ganz besonderes Themenheft. Nach 6 Jahren im Kreise der Mitherausgeber der Zeitschrift Der Radiologe werde ich zum Januar 2022 in den Kreis der Schriftleitung der RöFo wechseln. Dem Wissenschaftlichen Beirat der Zeitschrift Der Radiologe werde ich weiterhin angehören. Ich möchte mich sehr bei der Schriftleitung, den Professoren Christian Herold, Stefan Delorme, Thomas Helmberger und Wolfgang Reith, sowie den Ehrenherausgebern, den Professoren Gerhard van Kaick und Maximilian Reiser, für die stets sehr vertrauensvolle und durchweg sehr freundschaftliche Zusammenarbeit ganz herzlich bedanken. Mein großer Dank gilt des Weiteren Claudia Zappe und Sabrina Brecht vom Springer Verlag für die sehr angenehme Zusammenarbeit. Für die Zeitschrift Der Radiologe wird keine Lücke entstehen, denn mit Prof. Ulrike Attenberger wurde der Kreis der Schriftleitung bereits überaus kompetent nachbesetzt. Ich bin sehr zuversichtlich, dass diese von mir sehr geschätzte Zeitschrift weiterhin reüssieren wird.

Dieses Themenheft wird eingeleitet von einem Beitrag von Prof. Jürgen Freyschmidt aus Bremen zum Thema "Granulomatöse Skelettveränderungen", die sich durch Nachweis von multiplen osteolytischen oder osteosklerotischen Herden im Skelett äußern können. Auf Grund ihrer Seltenheit ist es bei der differenzialdiagnostischen Abklärung wichtig, auch an diese seltenen Erkrankungen wie die Langerhans-Zell-Histiozytose, die Skelettsarkoidose, die Mastozytose sowie die Lipoidgranulomatose zu denken. Es folgt ein Beitrag von Dr. Johanna
Luitjens und Prof. Andrea Baur-Melnyk aus München zu Skelettveränderungen bei hämatologischen Erkrankungen. Die Autorinnen führen aus, dass Leukämien, Anämien und chronische myeloproliferative Erkrankungen häufig zu einer Beteiligung des Skelettsystems führen. Auch in Deutschland muss differenzialdiagnostisch an Hämoglobinopathien wie die Sichelzellanämie und Thalassämien gedacht werden. Der nächste Beitrag von Prof. Tim Weber und Dr. Theresa Mokry aus Heidelberg behandelt Skelettveränderungen bei Plasmazelldyskrasien wie der monoklonalen Gammopathie unklarer Signifikanz, dem schwelenden und dem symptomatischen multiplen Myelom. Hier ist die Ganzkörper-Computertomographie (CT) inzwischen zur primär eingesetzten Bildgebungsmodalität in Deutschland geworden, insbesondere bei der symptomatischen Form. Es schließt sich ein Beitrag von Prof. Markus Uhl aus Freiburg zu den muskuloskeletalen Manifestationsformen der Neurofibromatose Typ I und der tuberösen Sklerose an. Die häufigere Neurofibromatose Typ I (Morbus von Recklinghausen) kann eine Reihe von Skelett- und Weichteilmanifestationen haben. Neurofibrome, diffuse und plexiforme Neurofibrome, maligne periphere Nervenscheidentumoren können beobachtet werden; Kyphosen und Skoliosen werden in etwa $20 \%$ der Fälle beobachtet. Bei der tuberösen Sklerose (Morbus Bourneville) finden sich am Skelett umschriebene Osteosklerosen, ähnlich Kompaktainseln im Markraum von Röhren- oder Plattknochen.

Der nächste Beitrag unter der Federführung von Dr. Daniela Kildal aus Brig/ Schweiz hat den Titel „Sklerosierende und 
hyperostotische Skelettveränderungen“.'In ihrem Beitrag werden u.a. die radiologischen Erscheinungsformen der Osteopetrose, Osteopoikilie, der Osteopathia striata sowie die Melorheosthose diskutiert. Es folgt ein Beitrag unter der Ägide von PD Dr. Carl Neuerburg aus München zum Thema "Systemische Skeletterkrankungen fragiler Knochen mit Fokus auf Frakturen“. Fragilitätsfrakturen, klassisch im Bereich des proximalen Humerus, des distalen Radius, der Wirbelsäule, des Beckens und der Hüften, treten oft bei betagten Patienten auf, z.B. aufgrund eines Stolperns. Moderne Diagnostikmethoden dieser Frakturen inklusive der Dual-EnergyCT und anderer Techniken zur quantitativen Knochendichtebestimmung werden dargestellt. Das Themenheft schließt ab mit einem Beitrag von Dr. Jürgen Wiens aus Wolfsburg zum Thema "Skelettveränderungen bei Knochenmetastasen: Morphologie und ausgewählte Differenzialdiagnostik". Auf Grund der Häufigkeit und der therapeutischen Konsequenz hat die Bildgebung einen sehr hohen Stellenwert, und Dr. Wiens stellt verschiedene radiologische Manifestationsformen und Morphologien von Knochenmetastasen dar. Die Radiologie hat hierbei die Möglichkeit, durch eine bildgesteuerte Biopsie auch die definitive Diagnose herbeizuführen.

Ich hoffe, liebe Leserinnen und Leser, Ihnen auch mit dem letzten Themenheft des Jahres 2021 zu diesem speziellen Thema der systemischen Skeletterkrankungen eine gute Zusammenfassung mit hoher Praxisrelevanz gegeben zu haben. Den Autoren danke ich ganz herzlich für ihre hervorragenden Beiträge. Ich wünsche Ihnen viel Spaß beim Lesen und hoffe, Sie befinden diese Zusammenstellung als interessant und hilfreich für Ihre praktische Arbeit. Mit den allerbesten Grüßen an Sie, liebe Leserinnen und Leser, und meinen besten Wünschen für die Zeitschrift Der Radiologe sowie mit meinen lieben Grüßen an die Schriftleitung und das Team des Springer Verlags,

Ihr

Marc-André Weber
Korrespondenzadresse

Prof. Dr. Marc-André Weber, M.Sc. Institut für Diagnostische und Interventionelle Radiologie, Kinder- und Neuroradiologie, Universitätsmedizin Rostock

Ernst-Heydemann-Str. 6, 18057 Rostock, Deutschland

marc-andre.weber@med.uni-rostock.de

Interessenkonflikt. M.-A. Weber gibt an, dass kein Interessenkonflikt besteht.

\section{Mini-Implantate}

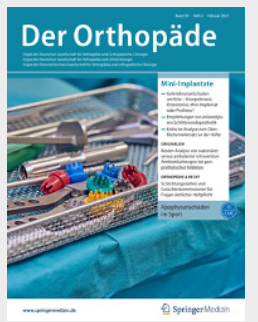

In den letzten Jahren haben sich viele neue Optionen zur Behandlung von Gelenkknorpelschäden oder der Arthrose ergeben. Doch problematisch bleibt der aktive Patient mit ausgereizter konservativer Terapie und fehlender Eignung für biologischen Gelenkerhalt oder vollständigen Gelenkersatz. Mini-Implantate bieten seit mittlerweile über 15 Jahren eine Option als Brückentechnologie, sind aber auch eine valide Option mit schneller Rehabilitationszeit bei schwierigen, lokalisierten Primärdefekten und Revisionssituationen.

Lesen Sie im Themenheft "Mini-Implantate" (Ausgabe 02/2021) von Der Orthopäde mehr zu folgenden Themen:

- Leitfaden unikompartimenteller Gelenkknorpelschaden am Knie

- Mini-Implantate am Fuß- und Sprunggelenk

- Empfehlungen zur unikondylären Schlittenendoprothetik im Wandel der Zeit

- Shoulder resurfacing for treatment of focal osteochondral defects to diff use osteoarthritis

- Neue Technologien in der unikondylären Endoprothetik - Pro und Contra

- Patellofemorale Inlay Implantate - ein Fortschritt in der patellofemoralen Endoprothetik?

Suchen Sie noch mehr zum Thema? Mit e.Med - den maßgeschneiderten Fortbildungsabos von Springer Medizin - haben Sie Zugriff auf alle Inhalte von SpringerMedizin.de. Sie können schnell und komfortabel in den für Sie relevanten Zeitschriften recherchieren und auf alle Inhalte im Volltext zugreifen.

Weitere Infos zu e.Med finden Sie auf springermedizin.de unter "Abos" 
Hier steht eine Anzeige.

黑 Springer 